\title{
Targeted gene disruption in Xenopus laevis using CRISPR/Cas9
}

\author{
Fengqin Wang ${ }^{1,3+}$, Zhaoying Shi ${ }^{2 \dagger}$, Yan Cui ${ }^{3,5}$, Xiaogang Guo ${ }^{3}$, Yun-Bo Shi ${ }^{4}$ and Yonglong Chen ${ }^{2,3^{*}}$
}

\section{Dear Editor}

To test if the CRISPR/Cas9 system can mediate targeted gene disruption in Xenopus laevis, we targeted ptf1a/p48 and tyrosinase in this species and found that in addition to high sequence disruption efficiency, clear phenotypes were observed in G0 embryos. ptf1a/ p48-targeted X. laevis embryos can mimic Xenopus tropicalis $p t f 1 a / p 48$-null mutant tadpoles with respect to the loss of pdip expression. Simultaneous disruption of two X. laevis tyrosinase homeologs leads to almost full albinism. Our data indicate that CRISPR/ Cas9 system is a simple and efficient tool for targeted gene disruption in $X$. laevis. It can be used for phenotype analysis in $\mathrm{G} 0$ embryos.

Since its introduction to modern biology in the 1950s, $X$. laevis has played a central role in most disciplines of biomedical research including developmental biology, biochemistry, and molecular biology [1]. Its allotetraploid genome represents a common polyploidy in amphibians, which challenges genetic studies on this species [2]. Fortunately, by the mid 1990s, X. tropicalis, the only diploid species in the Xenopus genus, was adopted as a genetically tractable complement to the classic model $X$. laevis. Together, the two frog species provide a unique and powerful system for addressing genome duplication/evolution, functional genomics, and human disease modelling at the post-genomic era [1].

Gene knockdown in X. laevis has been largely dependent on antisense Morpholino oligomers since 2000, which suffers from some off-target effects [3]. A recent systematic analysis reveals poor phenotypic correlation between published Morpholino-induced morphants and mutant lines in zebrafish [4], further highlighting the

\footnotetext{
* Correspondence: chenyl@sustc.edu.cn

${ }^{\dagger}$ Equal contributors

${ }^{2}$ Department of Biology, Shenzhen Key Laboratory of Cell Microenvironment, South University of Science and Technology of China, Shenzhen 518055, China ${ }^{3}$ CAS Key Laboratory of Regenerative Biology, South China Institute for Stem Cell Biology and Regenerative Medicine, Guangzhou Institutes of Biomedicine and Health, Chinese Academy of Sciences, Guangzhou 510530, China

Full list of author information is available at the end of the article
}

off-target effects of Morpholino oligomers. Transcription activator-like effector nuclease (TALEN) was shown effective for targeted gene disruption in X. laevis G0 embryos [1,5-7]. With the establishment of efficient targeted gene disruption in $X$. tropicalis using the clustered regularly interspaced short palindromic repeats (CRISPR) and CRISPR-associated 9 (CRISPR/Cas9) system [8], it is worth addressing if this simple method applies to $X$. laevis.

\section{CRISPR/Cas9 mediated disruption of $p t f 1 a / p 48$ in $X$. laevis} G0 embryos phenocopies $X$. tropicalis ptf1a/p48 mutants The basic helix-loop-helix transcription factor Ptfla/ p48 is a key regulator early for pancreatic precursor specification and late for exocrine pancreas formation $[9,10]$. Our previous studies indicate that targeted disruption of $X$. tropicalis ptf1a/p48 in G0 embryos using either TALENs [5] or CRISPR/Cas9 [8] leads to inhibition of pancreas formation as indicated by reduction of pancreas-specific marker gene pdip expression. These data are consistent with the $X$. laevis morphants [10] and Ptf1a/p 48 knockout mice [9]. Here, using our previously generated CRISPR/Cas9-targeted founder frogs, we established a $X$. tropicalis $p t f 1 a / p 48$ mutant line. Unlike Ptf1a/p48-null mutant mice that can survive whole gestation [9] likely due to mother supply of blood/nutrition and die at birth, the free-swimming $X$. tropicalis $p t f 1 a / p 48$ homozygous mutant tadpoles cannot develop to froglets and all died during feeding stages (around stages 47-48). Those collected at stage 43 showed complete absence of pdip expression (Figure 1A, C), while heterozygous tadpoles are indistinguishable from wild type siblings (Figure 1B, D), which is similar to the phenotype of Ptf1a/p48 knockout mice [9]. The mutant phenotype obtained here also verified the reliability of previous observations on X. laevis morphants [10].

Given the allotetraploid genome of $X$. laevis, every gene might have two pairs of homeologs. For ptf1a/ p 48 allele, only one cDNA sequence can be found 

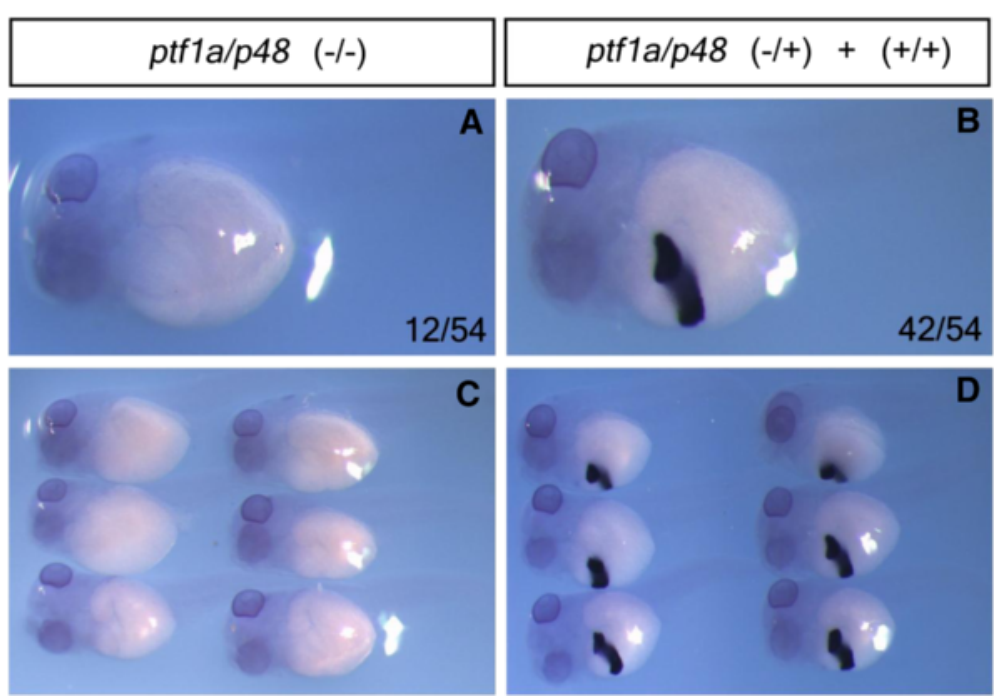

Figure 1 pdip expression is undetectable in X. tropicalis ptfla/p48-null mutant tadpoles. Whole-mount in situ hybridization analysis of pdip expression in $\mathrm{F} 2$ X. tropicalis ptf1a/p48 mutant tadpoles (stage 43). Ventral-lateral view, head to the left. (A, C) About 22\% homozygous tadpoles showed complete absence of pdip expression. (B, D) The remainder uniformly showed strong pdip expression, suggesting normal pancreas development in ptf1a/p48 heterozygous tadpoles.

$\begin{array}{llll}\text { ptf1a/p48-T1 } & & \\ \text { AGACGACTTTTTGGAAGACGATGTAGACTTCTTGGCCGGTCAGATCCAAGACTATTAC } & \mathbf{w t} & \\ \text { AGACGACTTTTTGGAAGACGATGTAGACCggacgatgtagaCTTGGCCGGTCAGATCC } & \mathbf{- 2 , + 1 3} & \\ \text { AGACGACTT---------------CTTGGCCGGTCAGATCCAAGACTATTAC } & \mathbf{- 2 1} & \\ \text { AGACGACTTTTTGGAAGACGATGTAGACTT---GGCCGGTCAGATCCAAGACTATTAC } & \mathbf{- 3} & \\ \text { AGACGACTTTTTGGAAGACGAT-TA--C---------------AGACTATTAC } & \mathbf{- 2 3} & \\ \text { AGACGACTTTTTGGAAGACGATGTAGACgatgtagtCTTGGCCGGTCAGATCCAAGAC } & \mathbf{- 2 , + 8} & \\ \text { AGACGACTTTTTGGAAGACGATGTAGA----tgtagatGTCAGATCCAAGACTATTAC } & \mathbf{- 1 1 , + 7} & \\ \text { AGACGACTTTTTGGAAGACGAT---------------CAGATCCAAGACTATTAC } & \mathbf{- 1 8} & \mathbf{\times 2}\end{array}$
AGACGACTTTTTGGAAGACGATGTAGACTTCTTGGCCGGTCAGATCCAAGACTATTAC

\section{ptf1a/p48-T2}

GGAGGCGGCTGAGGTCGGACGCGGAGATGCAGCAGCTCAGGCAGGCGGCGAATGTCCG GGAGGCGGCTGAGGTCGGACGCGGAGATGCAGCAGCTC-GGCAGGCGGCGAATGTCCG GGAGGCGGCTGAGGTCGGACGCGGAGATGCAGCAGCTC--GCAGGCGGCGAATGTCCG GGAGGCGGCTGAGGTCGGACGCGGAGATGCAGCAGAT---GCAGGCGGCGAATGTCCG GGAGGCGGCTGAGGTCGGACGCGGAGATGCAGgA----AGGCAGGCGGCGAATGTCCG GGAGGCGGCTGAGGTCGGACGCGGAGATGCAGCA------GCAGGCGGCGAATGTCCG GGAGGCGGCTGAGGTCGGACGCGGAGATGCAGCAG---------GCGGCGAATGTCCG GGAGGCGGCTGAGGTCGGACGCGGAGATGCAG--.--..---- GCGGCGAATGTCCG GGAGGCGGCTGAGGTCGGACGCGGAGATGCAGCAGCTC--.---.-----ATGTCCG

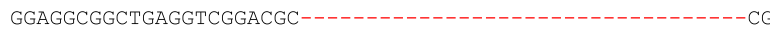
GG-_.

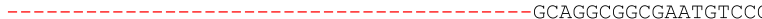
GGAGGCGGCTGAGGTCGGACGCGGAGATGCAGCAGCTCAGGCAGGCGGCGAATGTCCG

wt

$-1$ $-2$ $-3$ $-5,+1$ $-9 \times 2$ $-13$ $-34$ $-43$ $-57$

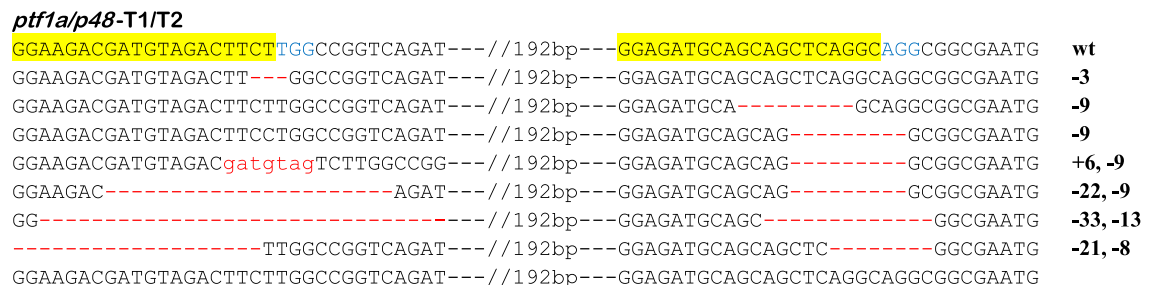

Figure 2 CRISPR/Cas9 is effective in targeting $X$. laevis ptf1a/p48. DNA sequencing data reveal the indel-inducing efficiencies of ptf1a/p48-T1 and ptf1a/p48-T2 in X. laevis embryos. For all panels, the wild-type sequence is shown at the top with the target site highlighted in yellow and the PAM sequence in blue. Red dashes indicate deletions (-) and lowercase letters in red indicate insertions (+). 

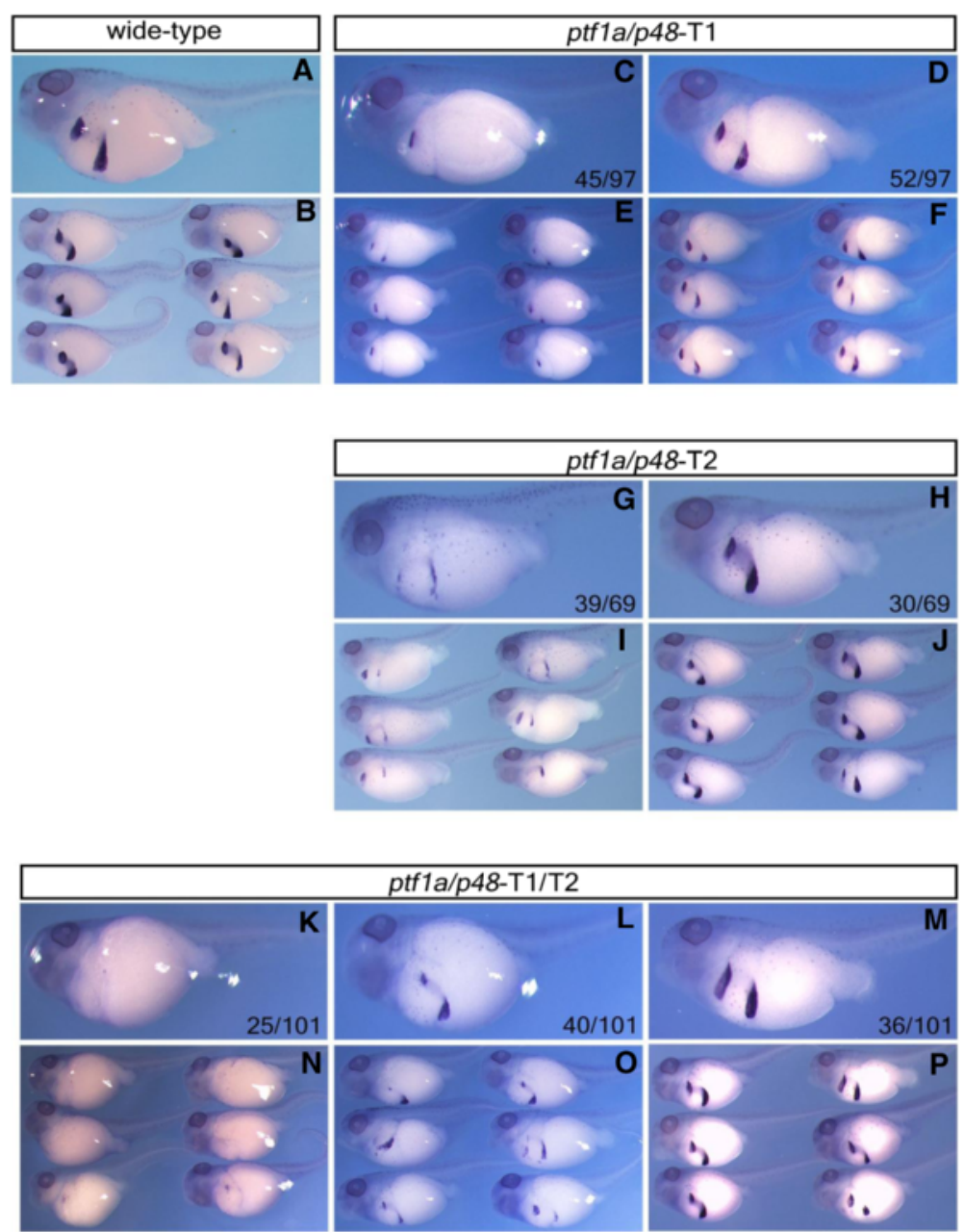

Figure 3 ptfla/p48-targeted X. laevis G0 tadpoles showed reduction of pdip expression. Whole-mount in situ hybridization analyses of pdip expression (stage 42). Ventral-lateral view, head to the left. (A, B) Wild type X. laevis tadpoles. (C, E) Severe reduction. (D, F) Mild reduction. (G, I) Severe reduction. (H, J) Mild reduction. (K, N) Almost complete absence. (L, O) Severe reduction. (M, P) Mild reduction.

from current databases (GenBank: DQ007931.1). We designed two gRNAs targeting the first exon of this $X$. laevis ptf1a/p48 locus, namely ptf1a/p48-T1 and ptf1a/p48-T2 (Additional file 1: Table S1). First we injected Cas9 mRNA together with these two gRNAs either alone or in combination into animal pole of 1-cell stage $X$. laevis embryos and assessed their indelinducing capacity via the $\mathrm{PCR} /$ sequencing protocol (Additional file 1: Table S2). Based on our experience with $X$. tropicalis embryos, we set the doses of both Cas9 mRNA and gRNAs at $300 \mathrm{pg} /$ embryo, which were proven optimal as on one hand efficient targeted gene disruption was obtained (Figure 2) and on the other hand no morphological malformation was observed in injected embryos. Overall, the targeted sequence disruption efficiency is above 50\% (Figure 2), which is comparable to that in $X$. tropicalis [8]. It should be noted that slightly more than $1 / 3$ of the disruption did not cause reading frame shift (Figure 2).

Next, we evaluated the pancreas development in injected embryos by whole mount in situ hybridization with a pdip probe. Similar to the observation in $p t f 1 a / p 48$-targeted $X$. tropicalis G0 embryos [8], severe inhibition of pdip expression was observed in all three injection groups (Figure 3). For reasons unknown, in spite of their lower sequence disruption efficiency in comparison to the individually injected embryos (Figure 2), ptf1a/p48-T1 and -T2 co-injected embryos displayed stronger pancreatic phenotype with quite a few of them showing almost complete absence of pdip expression reminiscent of $X$. tropicalis ptf1a/p48-null mutants (Figure 3K, N and Figure 1A, C). Taken together, these data indicate that CRISPR/Cas9 system is effective in X. laevis. For $p t f 1 a / p 48$, one gRNA is sufficient to cause an obvious pancreatic phenotype in $X$. laevis G0 embryos. 


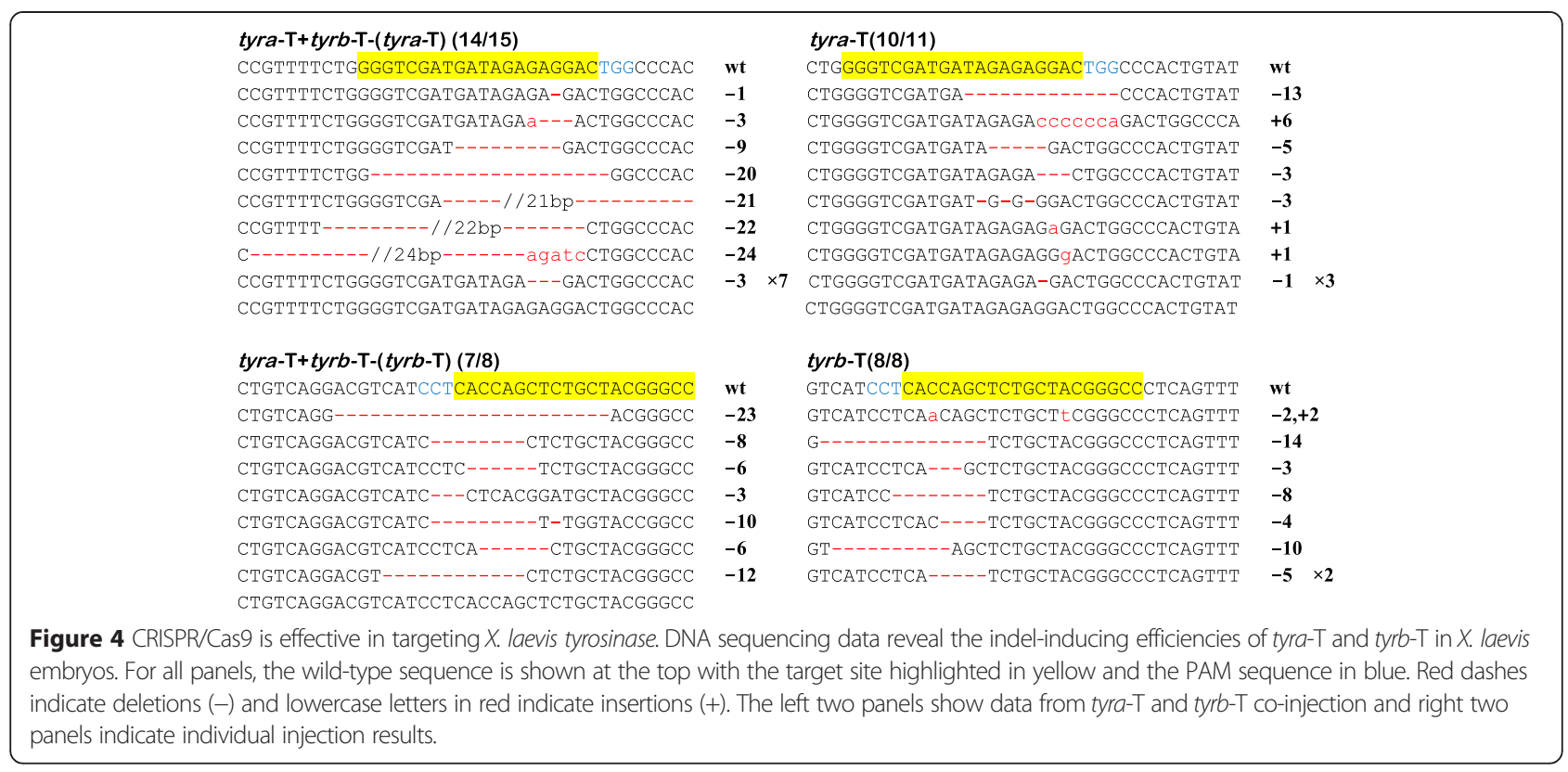
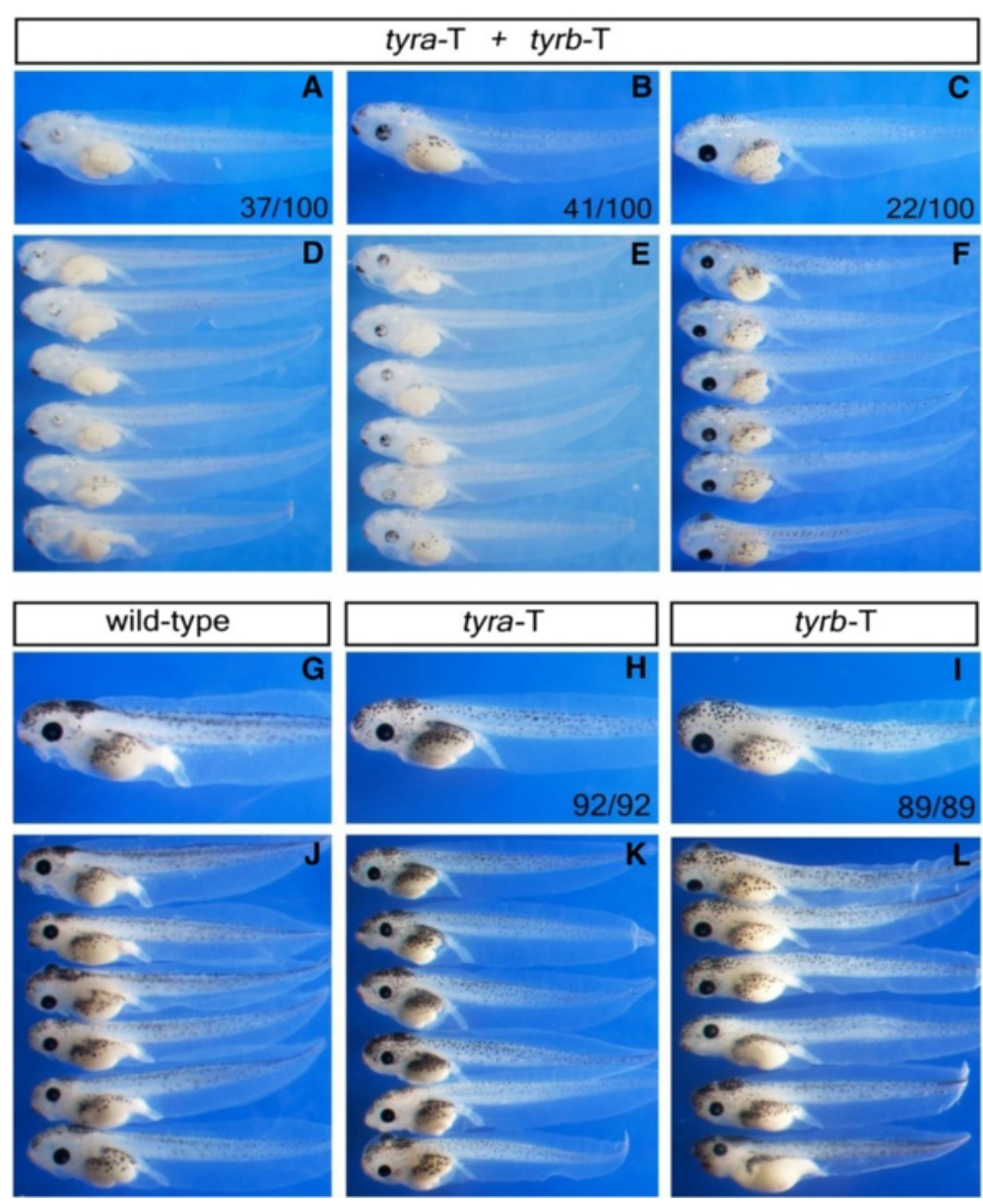

Figure 5 Simultaneous disruption of two X. laevis tyrosinase homeologs leads to albinism in G0 tadpoles (stage 42). Lateral view, head to the left. (A, D) Almost full albinism. (B, E) Severe inhibition of pigmentation. (C, F) Almost normal. (G, J) Wild type. (H, I, K, L) Almost normal. 


\section{Simultaneous disruption of two $X$. laevis tyrosinase homeologs leads to albinism}

To further test if CRISPR/Cas9 is a robust tool for gene targeting in $X$. laevis, we chose to target tyrosinase. There are two homeologs for $X$. laevis tyrosinase, tyra and tyrb [7]. A previous study showed that one pair of TALENs targeting a highly conserved region of the two homeologs was sufficient to induce albinism in $X$. laevis [6]. As no suitable gRNA targeting sites can be found in this conserved region for both homeologs, we have to design two gRNAs (tyra-T and tyrb-T) to target tyra and tyrb, respectively (Additional file 1: Table S1). Again, we injected Cas 9 mRNA (300 pg/embryo) with these two gRNAs either alone or in combination (300 pg/embryo for each gRNA) into the animal pole of $X$. laevis fertilized eggs. PCR/sequencing data indicate that both gRNAs are very effective with targeted sequence disruption efficiencies from $87.5 \%$ up to $100 \%$ (Figure 4 ). The tyra-T and tyrb-T co-injected tadpoles showed severe reduction of pigmentation (Figure 5B, E), with 37\% (37/ 100 ) of them showing almost full albinism (Figure $5 \mathrm{~A}$, D), which is stronger than TALENs-induced phenotype in $X$. laevis [6,7] and CRISPR/Cas9-induced phenotype in $X$. tropicalis [8]. An independent injection led to similar results. In contrast, although the sequence disruption efficiency remained high (Figure 4), individual injection of either tyra-T or tyrb-T did not cause any obvious alteration on embryonic pigmentation (Figure $5 \mathrm{H}, \mathrm{I}, \mathrm{K}, \mathrm{L}$ ), indicating the functional redundancy of tyra and tyrb. In sum, these data further confirm that CRISPR/Cas9 system is effective in $X$. laevis. Distinct homeologs of $X$. laevis genes can be easily disrupted by application of two gRNAs.

Due to Morpholino's potential off-target effects, a recent study recommends mutant phenotypes as the standard metric to define gene function in zebrafish [4]. Our previous study did not detect any CRISPR/Cas9-induced offtarget effects in $X$. tropicalis [8]. The issue of CRISPR/ Cas9's off-target effects in X. laevis remains to be defined. Nevertheless, our data demonstrate that CRISPR/Cas9 is equally a superb tool for targeted gene disruption in $X$. laevis as in $X$. tropicalis. It can be used for an immediate phenotype evaluation in $X$. laevis G0 embryos. It should be noted that the allotetraploid genome and longer generation time (1-2 years) of $X$. laevis makes it impractical to do genetic research. For establishment of stable knockout lines, we recommend to use the diploid frog $X$. tropicalis that has much shorter generation time (4-6 months).

\section{Additional file}

Additional file 1: Table S1. Oligonucleotides used to construct gRNA expression templates. Table S2. PCR primers used to amplify the targeted loci.
Competing interests

The authors declare that they have no competing interests.

\section{Authors' contributions}

FW, YS, and YChen designed the work and analyzed the data. FW, ZS, YCui, and XG carried out the experiments. YChen and FW wrote the manuscript. All authors read and approved the final manuscript.

\section{Acknowledgements}

This work was supported in part by National Basic Research Program of China (2015CB942803), the National Natural Science Foundation of China (31471367, 31271554, and 31301192), Shenzhen Key Laboratory of Cell Microenvironment (ZDSYS20140509142721429), and the Key Laboratory of Regenerative Biology, Guangzhou Institutes of Biomedicine and Health, Chinese Academy of Sciences. This research was also supported in part by the Intramural Research Program of $\mathrm{NICHD}, \mathrm{NIH}$.

\section{Author details}

${ }^{1}$ School of Life Sciences, Anhui University, Hefei 230601, China. ${ }^{2}$ Department of Biology, Shenzhen Key Laboratory of Cell Microenvironment, South University of Science and Technology of China, Shenzhen 518055, China. ${ }^{3}$ CAS Key Laboratory of Regenerative Biology, South China Institute for Stem Cell Biology and Regenerative Medicine, Guangzhou Institutes of Biomedicine and Health, Chinese Academy of Sciences, Guangzhou 510530, China. ${ }^{4}$ Section on Molecular Morphogenesis, Program in Cellular Regulation and Metabolism, Eunice Kennedy Shriver National Institute of Child Health and Human Development (NICHD), U.S. National Institutes of Health, Bethesda, Maryland, USA. ${ }^{5}$ University of Chinese Academy of Sciences, Beijing 100049, China.

Received: 23 February 2015 Accepted: 25 March 2015

Published online: 14 April 2015

\section{References}

1. Schmitt SM, Gull M, Brändli AW. Engineering Xenopus embryos for phenotypic drug discovery screening. Adv Drug Deliv Rev. 2014;69:225-46.

2. Tinsley RC, Kobel HR. Allopolyploid speciation. In: Tinsley RC, Kobel HR, editors. The Biology of Xenopus. Oxford: Clarendon Press; 1996. p. 391-402.

3. Eisen JS, Smith JC. Controlling morpholino experiments: don't stop making antisense. Development. 2008;135:1735-43.

4. Kok FO, Shin M, Ni CW, Gupta A, Grosse AS, van Impel A, et al. Reverse genetic screening reveals poor correlation between Morpholino-Induced and mutant phenotypes in Zebrafish. Dev Cell. 2015;32:97-108.

5. Lei $Y$, Guo X, Deng Y, Chen Y, Zhao H. Generation of gene disruptions by transcription activator-like effector nucleases (TALENs) in Xenopus tropicalis embryos. Cell Biosci. 2013;3:21.

6. Suzuki KT, Isoyama Y, Kashiwagi K, Sakuma T, Ochiai H, Sakamoto N, et al. High efficiency TALENs enable FO functional analysis by targeted gene disruption in Xenopus laevis embryos. Biol Open. 2013;2:448-52.

7. Sakane Y, Sakuma T, Kashiwagi K, Kashiwagi A, Yamamoto T, Suzuki KT. Targeted mutagenesis of multiple and paralogous genes in Xenopus laevis using two pairs of transcription activator-like effector nucleases. Dev Growth Differ. 2014;56:108-14.

8. Guo X, Zhang T, Hu Z, Zhang Y, Shi Z, Wang Q, et al. Efficient RNA/Cas9-mediated genome editing in Xenopus tropicalis. Development. 2014;141:707-14.

9. Krapp A, Knöfler M, Ledermann B, Bürki K, Berney C, Zoerkler N, et al. The bHLH protein PTF1-p48 is essential for the formation of the exocrine and the correct spatial organization of the endocrine pancreas. Genes Dev. 1998;12:3752-63.

10. Afelik $\mathrm{S}, \mathrm{Chen} \mathrm{Y}$, Pieler T. Combined ectopic expression of Pdx1 and Ptf1a/ p48 results in the stable conversion of posterior endoderm into endocrine and exocrine pancreatic tissue. Genes Dev. 2006;20:1441-6. 